\title{
Random magnets and correlations of stock price fluctuations
}

\author{
Bernd Rosenow $^{\mathrm{a}, *}$, Parameswaran Gopikrishnan ${ }^{\mathrm{b}, \mathrm{c}}$, \\ Vasiliki Plerou ${ }^{\mathrm{b}}$, H. Eugene Stanley ${ }^{\mathrm{b}}$ \\ a Institut für Theoretische Physik, Universität zu Köln, D-50937 Köln, Germany \\ ${ }^{\mathrm{b}}$ Center for Polymer Studies and Department of Physics, Boston University, Boston, MA 02215, USA \\ ${ }^{\mathrm{c}}$ Goldman Sachs, New York, USA
}

\begin{abstract}
Random magnets provide a paradigm for the study of competing interactions and frustration in physics. Here, we suggest that this paradigm is also useful for the study and explanation of correlations between stock price changes of different companies: it (i) provides for a mechanism to explain the origin of correlations, (ii) allows to understand the occurrence of power-law correlations in the time series of highly correlated eigenmodes, and (iii) is a useful framework for the analysis of optimal investment strategies where the knowledge of (anti-)correlations is an important prerequisite for the reduction of risk. (C) 2002 Elsevier Science B.V. All rights reserved.
\end{abstract}

PACS: 05.45.Tp; 89.90.+n; 05.40.-a; 75.10.Nr

Keywords: Random Magnets; Cross-correlations; Econophysics

The theoretical analysis of a physical system often starts by setting up a Hamiltonian describing both the dynamics of the basic constituents and the interactions between them. Then, one proceeds to calculate correlation functions relating physical observables to the microscopic Hamiltonian and compares them to experimental data. This procedure is possible because one has an a priori idea about the description of the system. The situation is different when describing economical systems, as there exists no microscopic theory which is applicable to all types of systems. For this reason, an inductive procedure starting with the analysis of empirical correlation function is more adequate. After getting an intuition for the system under consideration, one can proceed

${ }^{*}$ Corresponding author.

E-mail address: rosenow@thp.uni-koeln.de (B. Rosenow). 
to construct an effective theory which is able to reproduce the empirical findings and make additional predictions, which can be checked empirically.

In recent years, physics methods have been successfully applied to the study of financial markets [1-4]. In this paper, we suggest that cross-correlations between the price fluctuations of different stocks can be explained in terms of a magnetic model. Using methods of random matrix theory $[5,6]$ to estimate the coupling strengths of the magnetic model from the empirically measured correlations, we find that the interactions are predominantly ferromagnetic with a long-range part and several cluster components. In a random magnet picture, the long-range interaction gives rise to correlated fluctuations of the whole market, whereas the cluster interactions describe common fluctuations of stocks from the same business sector. In the problem of finding an investment in the stock market which exposes the invested capital to a minimum level of risk only, the expected returns of individual stocks add the complication of local random fields to the physics of ferromagnetic clusters.

The subject of our study are cross-correlations in the stock market. In the empirical part, we analyze stock prize changes of the 1000 largest publicly traded US companies for the years 1994/1995. The data of these transactions are recorded in the Trades and Quotes (TAQ) database published by the New York stock exchange. ${ }^{1}$ From the time series of prices $S_{i}(t)$ of stock $i, i=1 \ldots 1000$, we calculate the returns $G_{i}(t)=$ $\ln S_{i}(t+\Delta t)-\ln S_{i}(t)$. The time difference $\Delta t$ is chosen as $30 \mathrm{~min}$ to ensure that there is sufficient trading activity in the time interval on the one hand and that the empirical time series are sufficiently long on the other hand. Equal time correlations between returns of different stocks are measured by the cross-correlation matrix $\mathbf{C}$ with elements

$$
C_{i j}=\frac{\left\langle G_{i} G_{j}\right\rangle-\left\langle G_{i}\right\rangle\left\langle G_{j}\right\rangle}{\sqrt{\left\langle G_{i}^{2}\right\rangle-\left\langle G_{i}\right\rangle^{2}} \sqrt{\left\langle G_{j}^{2}\right\rangle-\left\langle G_{j}\right\rangle^{2}}} .
$$

In analogy to the analysis of correlation functions in physical systems, we study the eigenvalues and eigenvectors of $\mathbf{C}$ to make the huge amount of information $\left(5 \times 10^{5}\right.$ independent elements) more accessible. We diagonalize $\mathbf{C}$, rank order its eigenvalues $\lambda_{i}$ such that $\lambda_{k}<\lambda_{k+1}$, and denote the corresponding eigenvectors by $\mathbf{u}^{k}$. The bulk of the eigenvalues are smaller than two, their statistics is well described by random matrix theory (RMT) [7-13]. The agreement of most of the eigenvalues and eigenvectors with RMT predictions implies that they do not contain information and should be discarded when analyzing the information content of $\mathbf{C}$. For this reason, we look for correlations captured by eigenvectors with corresponding eigenvalues larger than the RMT bound. An analysis of the components of these deviating eigenvectors shows that they describe the influence of collective fluctuations of the whole market, of companies with a large

\footnotetext{
${ }^{1}$ Analyzed are data taken from the Trades and Quotes database published by the New York Stock Exchange, for the 1-year period 1994, recorded at $30 \mathrm{~min}$ intervals. Only those companies that survive the entire period are considered in our analysis.
} 
market capitalization, of companies in the same business sector, and of companies with business activities in the same geographic region [9].

Can financial correlations described by a model of interacting stocks? A generic physics model for the description of interacting particles is the soft spin model. Here, the fundamental spin variables are the instantaneous returns $g_{i}(t)=\tau_{0}(\mathrm{~d} / \mathrm{d} t) \ln S_{i}(t)$, which describe price changes between successive price ticks on the microscopic time scale $\tau_{0}$. The dynamics of the price changes $g_{i}(t)$ is governed by a system of coupled differential equations [9]

$$
\tau_{0} \frac{\mathrm{d}}{\mathrm{d} t} g_{i}(t)=-r_{i} g_{i}(t)-\kappa g_{i}^{3}(t)+\sum_{j} J_{i j} g_{j}(t)+\xi_{i}(t) .
$$

The noise terms $\xi_{i}(t)$ have correlators $\left\langle\xi_{i}(t) \xi_{j}\left(t^{\prime}\right)\right\rangle=\delta_{i j} \tau_{0} \delta\left(t-t^{\prime}\right)$, the term proportional to $r_{i}$ describes diffusion of returns, the interaction term with couplings $J_{i j}$ gives rise to correlations between returns of different stocks, and the cubic term is included to stabilize the theory in the vicinity of a critical point. To describe the fat tails of empirically observed distributions of price changes in our model, we must allow that the $\left\{r_{i}\right\}$ change with time and introduce multiplicative noise in the stochastic differential equation [14].

Starting from Eq. (2), we calculate cross-correlations of returns on a time scale $\Delta t \gg \tau_{0}$. In a weak coupling analysis under neglection of the cubic term in Eq. (2), one finds that the elements of the cross-correlation matrix $\mathbf{C}$ and of the coupling matrix $\mathbf{J}$ are related by

$$
C_{i j}=\delta_{i j}+J_{i j}\left(1 / r_{i}+1 / r_{j}\right)
$$

In this picture, the market correlations are caused by a long-range ferromagnetic coupling, and the industry sectors can be interpreted as local ferromagnetic clusters.

The soft spin model Eq. (2) can be used to relate the strength of a correlated mode to its relaxation time. One important result from the theory of critical phenomena is the observation that strong correlations are accompanied by long relaxation times. At a critical point, one even observes power-law correlations. For the special case of $r_{i} \equiv r$ and without the cubic coupling one can derive an analytic relation between an eigenvalue $\lambda_{i}$ of $\mathbf{C}$ and the relaxation time $\tau_{i}$ of the eigenmode $i$

$$
\lambda_{i}=N \tau_{i}^{2} / \sum_{i=1}^{N} \tau_{i}^{2}
$$

To check the prediction of slow relaxation for modes with large eigenvalues empirically, we associate a time series of returns $G^{(k)}(t)=\sum_{i=1}^{N} u_{i}^{k} G_{i}(t)$ with every eigenvector $\mathbf{u}^{k}$. The autocorrelation function for eigenmode $k$ is then given by $c^{(k)}(\tau)=\left\langle G^{(k)}(t+\right.$ $\left.\tau) G^{(k)}(t)\right\rangle$. We estimate the exponent of the correlation function by using the method of detrended fluctuation analysis (DFA) [15]. Empirically, we find power-law autocorrelations for the time series calculated from eigenvectors outside the random matrix bound (Fig. 1) [9]. The time series $G^{999}$ shows significant autocorrelations over several hours (Fig. 2). We consider the appearance of power law correlated eigenmodes as an 


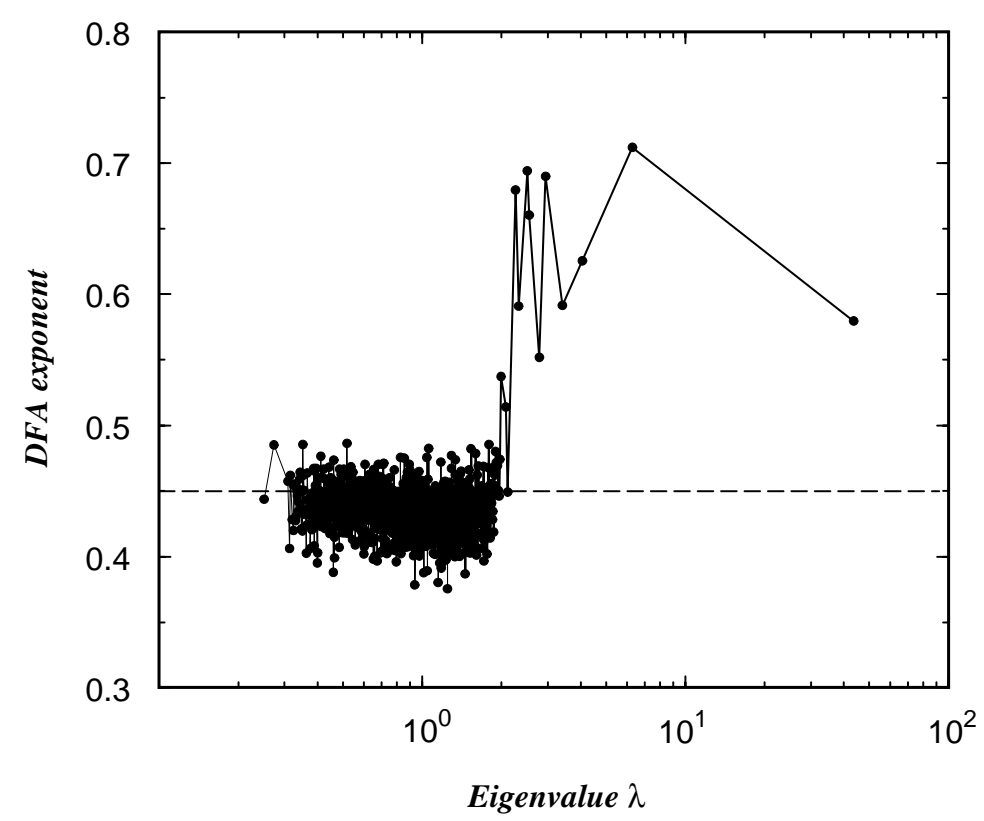

Fig. 1. Exponents of the detrended fluctuation function $F(\tau) \sim \tau^{v_{k}}$ for time series $G^{(k)}(t)$ defined by eigenvector $\mathbf{u}^{k}$ plotted against the eigenvalue $\lambda_{k}$. An exponent $v \approx 0.5$ indicates the absences of autocorrelation, whereas an exponent $v>0.5$ indicates autocorrelations decaying according to a power law $\tau^{-\gamma}$ with an exponent $\gamma=2-2 v$. For all eigenvectors, which are outside the RMT bound and can be identified with business sectors, we find exponents $v$ significantly larger than 0.5 indicating long-range power-law autocorrelations.

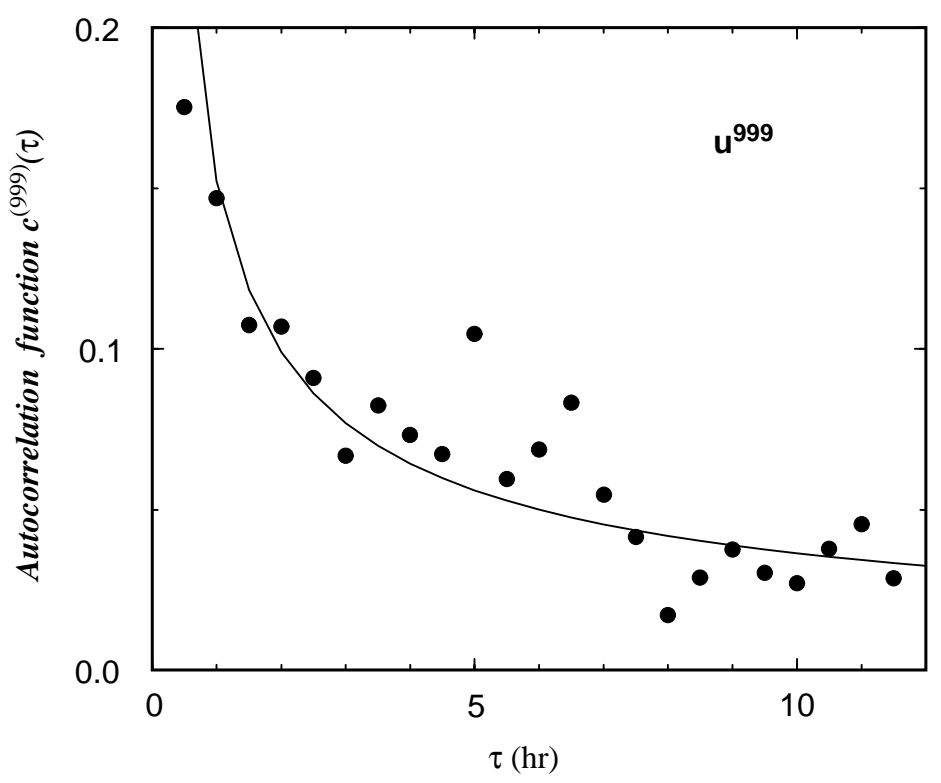

Fig. 2. Autocorrelation function $c^{(999)}(\tau)$ for the time series defined by the eigenvector $\mathbf{u}^{999}$. The solid line is a fit to a power law $c^{(999)}(\tau) \sim \tau^{-0.61}$. The error in determining the exponent is \pm 0.06 . 
indication of strong correlations in market subsectors and in that part of the market index $G^{(1000)}(t)$ which is not described by the S\&P500.

The knowledge of correlations between stock price changes is interesting not only from a fundamental point of view but also for practical applications. An investor in the stock market wants to expose the invested capital to a minimum level of risk only. Here, we measure the risk of an investment by the variance $D^{2}$ of its return $R$. The risk of an investment can be reduced by diversification, i.e., by investing not in one but in a portfolio of $N$ stocks. If stock prices were fluctuating completely uncorrelated, the risk of a diversified investment would decrease like $1 / \sqrt{N}$. In reality, stock price fluctuations are correlated and knowledge of the cross-correlation matrix $\mathbf{C}$ is necessary to calculate the risk of an investment. If one considers a simple situation, where short selling of stocks is allowed at no extra fee and where one invests into stocks only, the problem of calculating an investment with minimum risk for a given return is equivalent to minimize the free energy [16]

$$
F=\frac{1}{2} \sum_{i, j=1}^{N}\left(C_{i j} \sigma_{i} \sigma_{j}\right) m_{i} m_{j}-\mu \sum_{i=1}^{N} m_{i} R_{i}-h \sum_{i=1}^{N} m_{i}
$$

The first term on the r.h.s. of Eq. (5) describes the risk of an investment in the stock market with a fraction of money $m_{i}$ invested into stock $i$. The requirement of a fixed total return $R=\sum_{i}^{N} R_{i}$ is included into the minimization problem by using a Lagrange multiplier $\mu$, and the constraint of a fixed total capital is included by adding a term $h \sum_{i}^{N} m_{i}$.

In Eq. (5), the correlation matrix times volatilities is the coupling matrix in a mean-field magnetic model. As discussed in the last section, the dominant interaction is a long-range ferromagnetic coupling describing correlations in the whole market. In addition, there are interactions between clusters of companies from the same business sector. The constraint of a fixed total return gives rise to a random field term in Eq. (5). From this point of view, the calculation of an optimal portfolio is equivalent to calculating the mean field solution for a random field ferromagnet.

In [9,17-19] RMT techniques were applied to the problem of forecasting correlations as an input for portfolio construction. In [19] it was shown that portfolios constructed using just the ferromagnetic coupling and the local clusters have a lower risk than portfolios calculated by using the empirical correlation matrix or the standard method of including just the market index as a source of correlations.

The problem of finding an optimal investment in futures markets is more complicated because there the investor must make a deposit for all transactions. Therefor, the linear constraint of fixed total capital in Eq. (5) must be replaced by a nonlinear constraint $\sum_{i=1}^{N}\left|m_{i}\right|=1$. With the nonlinear constraint, the portfolio problem is equivalent to finding the ground state of a spin system instead of the mean field solution. If one uses the empirical cross-correlation matrix $\mathbf{C}$ to calculate the interaction matrix of the portfolio free energy, the optimization problem has a spin glass type complexity [20]. When keeping only the ferromagnetic couplings describing the market and cluster correlations, the complexity of the optimization problem is reduced that of a random field ferromagnet [19]. 
In summary, we have shown that the framework of magnetic systems is useful for describing and explaining cross-correlations between stock price fluctuations. The empirically observed market correlation can be related to a long-range ferromagnetic coupling in a spin model, whereas correlations within business sectors correspond to cluster interactions. Critical slowing down for strongly correlated modes as predicted by the spin model is observed in empirical data. Finally, we have argued that the problem of finding an optimal investment in the stock market is related to the solution of a random magnet problem in physics.

\section{Acknowledgements}

We thank J.-P. Bouchaud, L.A.N. Amaral, and D. Stauffer for interesting discussions, and the National Science Foundation for support.

\section{References}

[1] I. Kondor, J. Kertész (Eds.), Econophysics: An Emerging Science, Kluwer, Dordrecht, 1999.

[2] J.-P. Bouchaud, M. Potters, Theory of Financial Risk, Cambridge University Press, Cambridge, 2000.

[3] R.N. Mantegnam, H.E. Stanley, An Introduction to Econophysics, Cambridge University Press, Cambridge, 2000.

[4] J. Voit, The Statistical Mechanics of Financial Markets, Springer, Berlin, 2001.

[5] E.P. Wigner, Ann. Math. 53 (1951) 36.

[6] T. Guhr, A. Müller-Groeling, H.A. Weidenmüller, Phys. Rep. 299 (1998) 189.

[7] L. Laloux, P. Cizeau, J.-P. Bouchaud, M. Potters, Phys. Rev. Lett. 83 (1999) 1469.

[8] V. Plerou, P. Gopikrishnan, B. Rosenow, L.A.N. Amaral, H.E. Stanley, Phys. Rev. Lett. 83 (1999) 1471.

[9] P. Gopikrishnan, B. Rosenow, V. Plerou, H.E. Stanley, Phys. Rev. E 64 (2001) 035106(R); see also cond-mat/0011145.

[10] S. Drozdz, F. Gruemmer, F. Ruf, J. Speth, Physica A 287 (2000) 440.

[11] J.D. Noh, Phys. Rev. E 61 (2000) 5981.

[12] Z. Burda, J. Jurkiewicz, M.A. Nowak, G. Papp, I. Zahed, cond-mat/0103108.

[13] P. Gopikrishnan, V. Plerou, B. Rosenow, L.A.N. Amaral, T. Guhr, H.E. Stanley, Phys. Rev. E, in press (2002) cond-mat/0108023.

[14] H. Takayasu, A.-H. Sato, M. Takayasu, Phys. Rev. Lett. 79 (1997) 966.

[15] C.-K. Peng, S.V. Buldyrev, S. Havlin, M. Simons, H.E. Stanley, A.L. Goldberger, Phys. Rev. E 49 (1994) 1685.

[16] E.J. Elton, M.J. Gruber, Modern Portfolio Theory and Investment Analysis, Wiley, New York, 1995.

[17] L. Laloux, P. Cizeau, M. Potters, J.-P. Bouchaud, Int. J. Theor. Appl. Finance 3 (2000) 391.

[18] S. Pafka, I. Kondor, cond-mat/0111503.

[19] B. Rosenow, V. Plerou, P. Gopikrishnan, H.E. Stanley, Europhys. Lett., in press (2002) cond-mat/ 0111537.

[20] S. Galluccio, J.-P. Bouchaud, M. Potters, Physica A 259 (1998) 449. 